\title{
Sequence Validation Based Extraction of Named High Cardinality Entities
}

\author{
Khamisi Kalegele ${ }^{1}$, Hideyuki Takahashi ${ }^{1,2}$, Kazuto Sasai ${ }^{1,2}$, Gen Kitagata ${ }^{1,2}$, \\ Tetsuo Kinoshita ${ }^{1,2}$ \\ ${ }^{1}$ Graduate School of Information Sciences, Tohoku University, Sendai, Japan \\ ${ }^{2}$ Research Institute of Electrical Communication, Tohoku University, Sendai, Japan \\ Email: kalegs@k.riec.tohoku.ac.jp, kazuto@fir.riec.tohoku.ac.jp,minatsu@fir.riec.tohoku.ac.jp, \\ hideyuki@riec.tohoku.ac.jp, kino@riec.tohoku.ac.jp
}

Received May 21, 2012; revised July 26, 2012; accepted August 6, 2012

\begin{abstract}
One of the most useful Information Extraction (IE) solutions to Web information harnessing is Named Entity Recognition (NER). Hand-coded rule methods are still the best performers. These methods and statistical methods exploit Natural Language Processing (NLP) features and characteristics (e.g. Capitalization) to extract Named Entities (NE) like personal and company names. For entities with multiple sub-entities of higher cardinality (e.g. linux command, citation) and which are non-speech, these systems fail to deliver efficiently. Promising Machine Learning (ML) methods would require large amounts of training examples which are impossible to manually produce. We call these entities Named High Cardinality Entities (NHCEs). We propose a sequence validation based approach for the extraction and validation of NHCEs. In the approach, sub-entities of NHCE candidates are statistically and structurally characterized during top-down annotation process and guided to transformation into either value types (v-type) or user-defined types (u-type) using a ML model. Treated as sequences of sub-entities, NHCE candidates with transformed sub-entities are then validated (and subsequently labeled) using a series of validation operators. We present a case study to demonstrate the approach and show how it helps to bridge the gap between IE and Intelligent Systems (IS) through the use of transformed sub-entities in supervised learning.
\end{abstract}

Keywords: Entity Recognition; Supervised Learning; Sequence Validation; Intelligent Systems; Text Mining

\section{Introduction}

Web aggregated content has become so popular and useful that it is considered indispensable. While utilizing the information that Web content provides, users are also so busy populating new data into the Web. It is a cycle of sharing, searching, browsing, to mention some, that is undertaken by so many people in vast domains. Like many other stake holders in their respective disciplines of this cycle, scientists and IT professionals are playing a vital role in facilitating easy access to this information through many a field like Information Extraction (IE) and text mining. IE, particularly, involves extraction of specific and exact pieces of information extracts in addition to searching for documents which contain them. Examples of extracts for which IE systems have been proposed include citations [1,2], course descriptions [3], news items [4], protein data [5] etc. Within IE systems, one of the most vital solutions is Named Entity Recognition and Classification (NERC). A field which has been extensively researched over the past 20 years.

As described in [6,7], Named Entity (NE) is a term widely used in NPL and was designed such that the expression "Named" aims at restricting the task to only those entities for which one or many rigid designators stand for the referent. For instance, a Japanese national university located in Sendai city, Miyagi prefecture in the Tohoku Region, is referred to as Tohoku University. Over the years, many NE have been studied, from proper names $[2,8]$, email addresses to bioinformatics domain [5].

Successfully approaches to NERC have mostly employed hand-coded pattern rules and statistical methods with varying degrees of automation using Machine Learning (ML) techniques. The basis to these approaches is NLP related features like orthographic features (e.g. capitalization) and parts-of-speech (e.g. nouns). Ultimate reliance to NLP features have seen NERC limited to only those entities which are speech compliant and appear in well formed sentences. As a result, following our observations, most NERC systems have dealt with entities of up to few sub-entities of lower cardinality levels. For instances, with the exception of open sub-entities like 
personal names, there are limited instances of a title (e.g. Mr., Mrs., Dr. etc.). This fact, together with mostly fewer number of sub-entities, enables writing of handful handcoded pattern rules in many successful NERC approaches.

In the ongoing Web-facilitated data deluge (as referred to in [9]), we are seeing diverse efforts of harnessing Web information for which NERC does not suffice as a vital building block to IE. So, many entities are now in need to be recognized, classified and organized, in a way that NE have been, if the full potential of harnessing Web information for use in various domains is to be realized. Only that these many entities are neither speech compliant nor do they appear in well formed sentences. An example in network administration domain would include system commands like ssh and netstat commands etc. In ML, as another example, instances can also be considered an entity because for a particular learning task and environment the structure of an instance is fixed, e.g. ARFF format in WEKA [10].

In this paper we refer to the entities which are neither directly speech compliant nor do they appear in well formed sentences as Named High Cardinality Entities (NHCEs) and propose an approach for their extraction. Unlike NE, since NHCE has a fixed format and structure, it is necessary to conduct a validation test after extraction because a NHCE can be structurally correct but invalid. Therefore, our proposed approach does include not only extraction but validation as well. The employed validation method in our proposed approach is sequence based whereby NHCE candidates are regarded as sequences. Validation is conducted in two phases: during top-down extraction (using generic pattern rules) and in post-processing phase (statistical). The approach enables NHCE sub-entities to transform as more observations are made. This transformation is achieved using a ML-based transformation model.

In the past, an increase in online publications gave rise to research interests in classifying and organizing citation extracts. As a result, we have seen success in products like citation databases (Citeseer [11]) whose power in facilitating information sharing among researchers have now been unleashed. We believe that progress in recognition and classification of NHCEs will facilitate better organization of many other information entities in the Web like technical forums (technical forum databases), ML training data (training data databases) etc. This will also help in bridging the gap between IE systems and Intelligent Systems (IS) through areas like supervised learning. In this paper, we also present a case study and demonstrate how recognition and classification of NHCEs can help in reducing this gap.

The rest of this paper is organized as follows. We provided an overview to IE systems, and define and discuss
NHCE in the next Section 2. Our proposed approach is presented in Section 3 and a case study in Section 4. In Section 5, we discuss related works. Section 6 presents our future research direction and concludes this paper.

\section{Information Extraction}

\subsection{Overview and Our Contributions}

IE systems deal with extraction of pieces of information from text based on some predefined concepts. Four factors provide the dimensions for their presentation and discussion. These are source-document type, extracts type, involved techniques and automation degree.

1) Source-Document Type: IE System can be designed to deal with either unstructured $[1,5]$ or semi-structured $[3,8]$ or structured [12] or both sources of data. Extraction difficulty easies as document structuredness increases.

2) Extracts Type: In most cases, extracts are very specific to a system. Types of extracts for which systems have been designed in the past include single-entity extracts or NE like names (of places, of persons etc.), a pattern (multi-entity extracts e.g. publication citation [1, $2]$ ), entities relationship (substance-protein metabolization [5]), and concepts (e.g. document or sentence classification).

3) Techniques: The basic IE systems involve sentence splitting, tokenization, named entity recognition, syntactic parsing and pattern recognition. Variations are with the last three whereby some systems use lookup lists and dictionary/thesaurus [8], some use pattern and logical rules (e.g. using JAPE) and others use ML techniques and ontologies [4]. Non rule-based approaches are also referred to as statistical methods. They are characterized by their approach of using models to label entity tokens. Various models have been used including ordered classification, Hidden Markov Models, HMM [13,14], Conditional Markov Model, CMM [15], Conditional Random Fields, CRF [16] etc. CRF-based are currently regarded as state-of-the-art methods in assigning labels to either tokens or their sequences.

4) Automation: The degree of automation is inline with the used technique. With rule-based techniques, automation is in rule generation. With ML techniques, automation is in model creation for either classification or clustering or for rule generation. The central point in automation has been in how much of labeled documents is needed to initiate an extraction process. In ML-based apporaches, progress has been made from supervised learning [17] to semi-supervised learning [7] to unsupervised learning [18]. Despite tremendous achievements in automation, the most reliable and efficient of systems are still hand-coded ones. Although ML-based approaches have huge potentials, they still face a critical challenge 
when it comes to labeling of examples for their consumption.

Commonly, IE systems are designed under NL-dependence context. Few have ventured into NL-independence. In [15], a probabilistic tagging approach for tagging sequences of words constituting NEs, based on HMM and NL-independence, is proposed. This approach, however, considered only NE with few sub-entities of low cardinality like personal and organization names. The task of classifying extracts which conform to NHCE has been approached by few researchers [1]. Extracts which are commonly dealt with and used for demonstrations are citation extracts. To the best of our knowledge, most IE systems which have dealt with extracts which conform to NHCE have only focused on a parsing task using already annotated extracts [1].

IE field is still facing a good number of challenges. We have already highlighted two of the biggest challenges (labeling of examples and NHCE recognition and classification). Other challenges, as described in [19], are those beyond automation and scalability of systems. These includes domain adaptation and an intriguing problem to demonstrate extracts' utility to IS, to mention few.

Our focus in this paper is on recognition and classification of NHCEs. We believe that being able to recognize and classify NHCEs brings in another milestone towards improved utility of extracts. Our contributions which are put forward can be expressed from different points of view. One is in leveraging the Web when solving ML problems like classifying or associating items etc. Typically, these tasks require examples which are always painfully produced. For long ML community have longed for data processing tools and approaches which will enable them to exploit the abundant Web content. We believe that the proposal in this paper brings ML community closer to Web content leveraging. An- other point of view is in relation to limitation to speech compliant entities. Our work is part of the long term effort of organizing non-speech entities (e.g. source codes, ML training data, network administration forums) in order to improve their sharing and utility. In a conceptual diagram shown in Figure 1, the left side shows the current common practice and focus whereby ML and other methods are applied on Web content to further improve efficiency of IE systems in extraction process. The right side gives a picture of our contribution whereby the immediate beneficiary of the various processing, in addition to IE systems, is ML.

\subsection{Named High Cardinality Entity}

We use the term NHCE to refer to those atomic entities in text which contains sub-entities of high cardinality. Our expression "Named High Cardinality Entity" loosens the expression "Named Entity" by including not so rigid designators which the later require. NHCE is not limited to speech compliant entity atomicity. For instance, "proper names" as NEs can not be worked out outside speech context. NHCEs can be regarded as further loosening of temporal expressions type of NEs like money in order to include other entities like computer systems related atomic entities and ML related entities which are increasingly becoming popular. On one side, NHCE overlaps $\mathrm{NE}$ to include such entities like citations and ssh commands (computer systems related entities). On the other side, NHCE includes such temporal expressions like training data instances (ML related) which are not covered by NE.

Three aspects in which NHCE differs from NE are sub-entity cardinality, speech compliance and rigidness of designators. These are summarized in Table 1. Although not formally stated, researches in NERC have
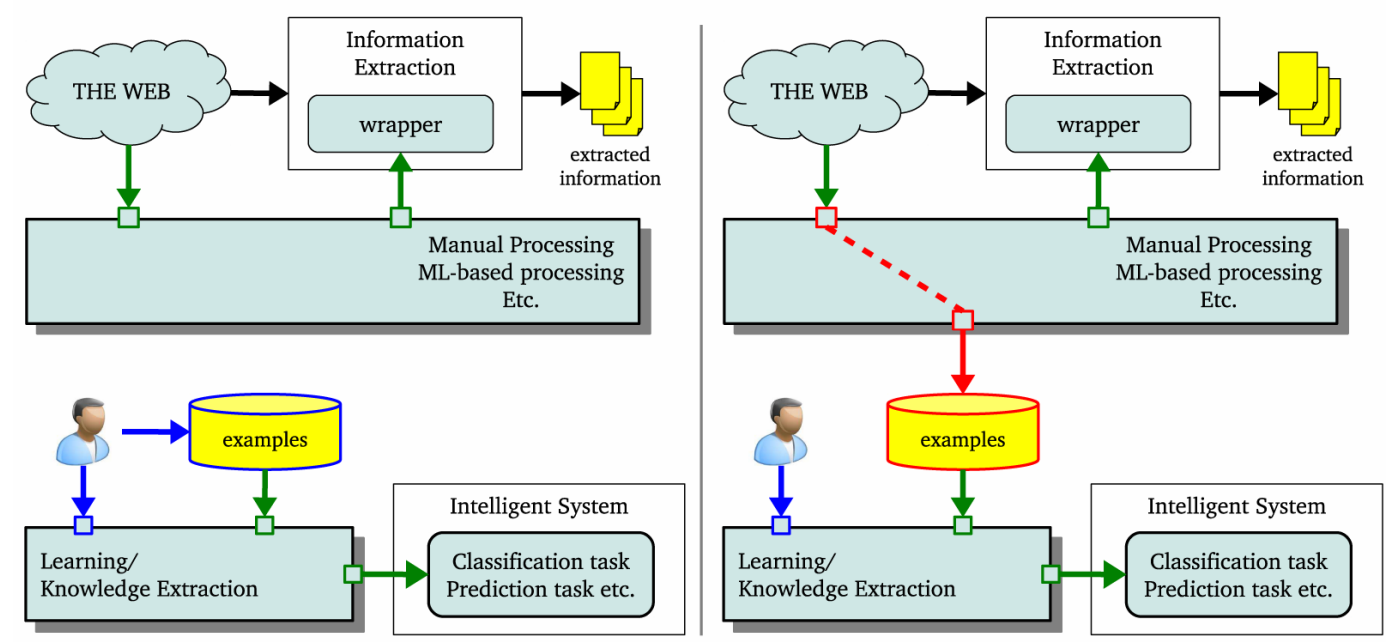

Figure 1. Conceptual diagram: Bridging the gap between IE and IS. 
Table 1. How NHCE differs from NE.

\begin{tabular}{lll}
\hline Aspect & NE & NHCE \\
\hline Sub-entity cardinality & Mostly low & Any level \\
Designator rigidness & Rigid & Any rigidness \\
Speech compliance & Compliant & Non-compliant \\
\hline
\end{tabular}

mostly dealt with relatively lower cardinality entities, e.g. the "enamex" (names of persons, locations etc.) and "timex" (date, time, money etc.). All these are speech compliant and constitute very rigid designators.

\section{The Proposed Approach}

\subsection{Problem Formulation}

Suppose an $n$-entity NHCE whereby each sub-entity is either a token or a NE referred to as a type. An entity field $f_{i}: 0<i \leq n$ within that NHCE can either be unoccupied (missing sub-entity) or occupied by any of the $\left\|T^{i}\right\|$ types $\left(t^{i} \in T^{i}\right)$ which are valid for that field. There are, therefore, $\prod_{i=1}^{n}\left(T^{i}+\phi_{i}\right)$ possible sequences of this $n$-entity NHCE. $\phi_{i}$ is either one or zero based on whether entity $n_{i}$ can be missing $\left(\phi_{i}=1\right)$ or not $\left(\phi_{i}=0\right)$.

For instance, a linux command can be considered as a 6-entity NHCE taking the format shown in Table 2. In an ssh command, valid type for ID field is only "ssh", i.e. 1 -cardinality. For OPTION field, there are 25 valid types

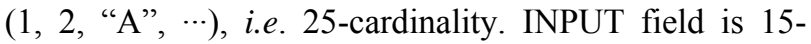
cardinality (ip, path, ․). ARGUMENT field is 2-cardinality (host and user). For COMMAND field, there are almost as many as all linux commands as valid types (more than 400). DESTINATION field is 0-cardinality. For simplicity, without considering COMMAND field, there are therefore at least 750 possible sequences of ssh command some of which are invalid. For example, in ssh command sequences above, although the second sequence is structurally correct, it is not a valid ssh command.

This paper addresses extraction and validation of $n$-entity NHCE so that its extracts can be used in solving ML problem by providing labeled training examples.

\subsection{Overview}

We propose a sequence validation based approach for the extraction and validation of NHCEs. Our approach is a top-down one in which generic pattern rules are used to extract text fragments that constitute the desired NHCE extracts. These text fragments are parsed into sub-entities and matched with pre-defined template sequences which are statistically scored using sequence frequency-inverse page frequency, sf-ipf (explained in Section 3.5). The sub-entities and the matched template sequences are then
Table 2. Example of $\boldsymbol{n}$-entity format.

\begin{tabular}{l}
\hline Format \\
ID OPTION INPUT ARGUMENT COMMAND DESTINATION \\
Examples: \\
ssh ? -R 99:1host:22 82:1host:22 ? ? \\
ssh -x -L 34:1host:66 server.com sleep 10? \\
mv? ? file1 ? file2 \\
Key: \\
? represents a missing entity \\
\hline
\end{tabular}

passed through a series of three categories of validation operators in which sub-entity sequences (extract sequences) are also labeled (valid or invalid) as described in Section 3.5. By characterizing sequence fields, the approach enables sub-entity occurrences to be either extended into value types (v-types) or transformed into either basic structural types (b-types) or user-defined types (u-types) using a ML-based transformation model. Finally, sub-entity transformed and labeled extract sequences of the desired NHCE are used to generate output for use in solving ML problems like validation or classification of the NHCE under consideration (can also be for use in further NHCE extraction). A schematic which summarizes the involved processes (template matching, entity transformation and validation) is shown in Figure 2. We explain the motivations and requirements which led to this approach designed in Section 3.3. It is inline with these requirements that the rest of the approach is presented.

\subsection{Requirements and Motivations}

1) Annotation and Parsing: The objective of leveraging Web content necessitates an annotation process for the identification of text fragments which constitute the desired NHCE and a wrapper for parsing those fragments into sub-entities. Instead of manual supply of documents (as text source or for training), an iterative and interactive extraction can allow us to leverage Web content and save human capital.

2) Validation of Sub-entity Sequences: Since knowledge about the extracts is initially not exact enough, a natural mechanism is to consider all sequences of sub-entities which constitute the desired NHCE extracts. To validate or invalidate an extracted text fragment and subsequently label it, a variety of means (e.g. statistical, expert input) is necessary if one is to expect comprehensive results.

3) Sub-entity Transformation: To cope with the evolving and transforming nature of Web information, subentities must be allowed and guided to transformation in order to accommodate new occurrences as more NHCE extracts are seen.

\subsection{Annotation and Parsing}

We adopt a top-down extraction process for annotating 


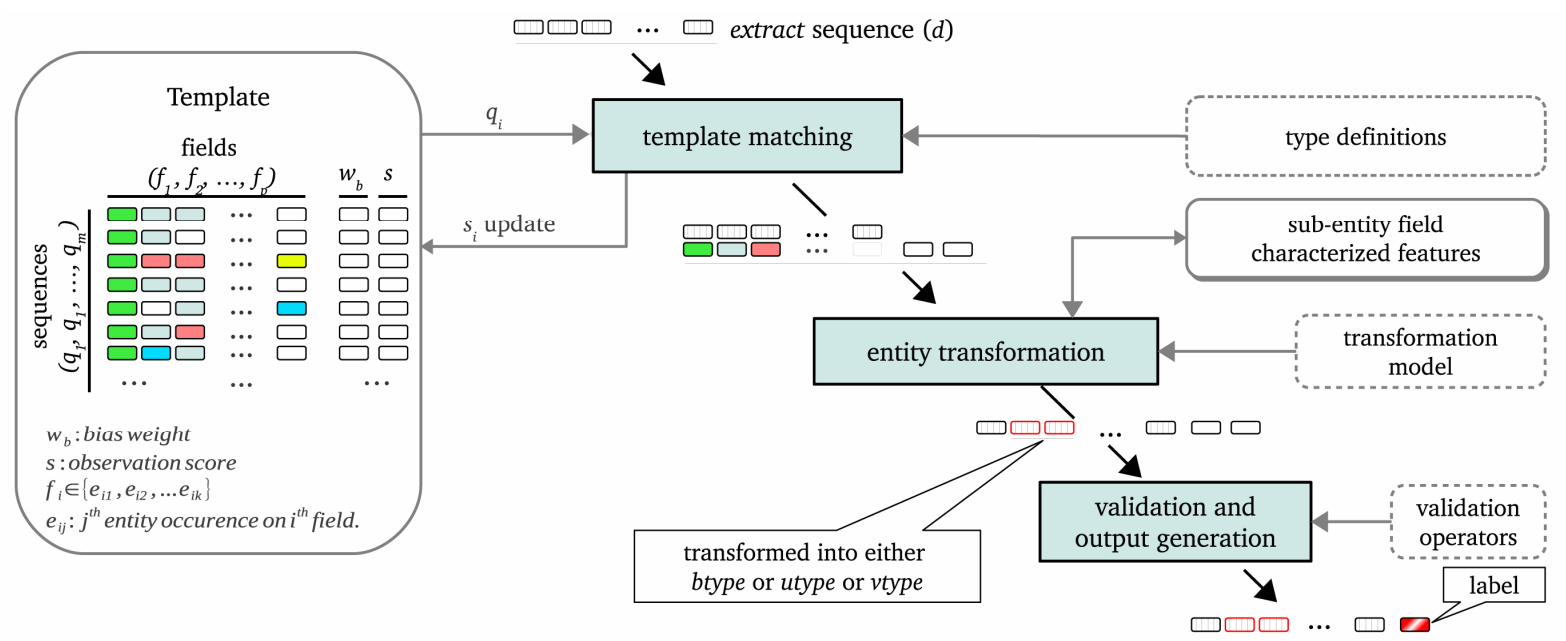

Figure 2. NHCE extraction process: A schematic diagram showing template matching, entity transformation and sequence validation.

and parsing extracts. Generic rules are used to extract text fragments which constitute the desired extract. At this stage, semantical and structural relationships are ignored. For instance, when ssh commands are desired, ssh $-R$ 5555: localhost: 3389 and ssh -l 5555: localhost: 3389 will all be extracted because the relationships between $-R,-l$ and 5555: localhost: 3389 are ignored. Shown in Figure 3 is the employed generic rules format in JAPE [20].

In the generic rule format, the terms ent $t_{i}$ are logical expressions for matching sub-entities. Under JAPE, these can be organized into JAPE macros. A combination of sub-entities forming up a sequence is bound to a label $l_{i}: 0<i \leq n$ which is used as a reference to the matched text fragment during post-processing after the rule has fired. For each label $l_{i}$, there are intermediate labels $l_{i j}: 0<j<i$ which are used for parsing the matched text fragment. This annotation approach, therefore, also serves as a parser for the desired NHCE extract.

We use rules in this initial stage and conduct statistical processing in later semi-automated stages because rules are easier to interpret, develop and deploy while statistical processing brings in robustness [21] to noise for unstructured data.

\subsection{Sequence Validation}

Extract sequences are then validated using template sequences and a set of two other categories of operators (ontological and direct expert input) as shown in Figure 4(a). Operators $T=\left(l_{t}, p_{t}\right), O=\left(l_{o}, p_{o}\right)$ and $R=\left(l_{r}, p_{r}\right)$ are such that a sequence $d_{i}$ (of objects $\left.o_{k}: 0<k \leq n\right)$ for which either $p_{t}\left(d_{i}\right)=$ true or $p_{o}\left(d_{i}\right)=$ true or $p_{r}\left(d_{i}\right)=$ true is labeled either $l_{t}$ or $l_{o}$ or $l_{r}$ respectively. Operators serve to bring flexibility into validation process and enables hands-on by an

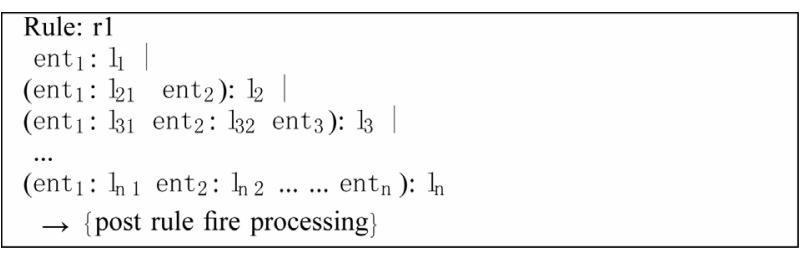

Figure 3. Pattern rule format.

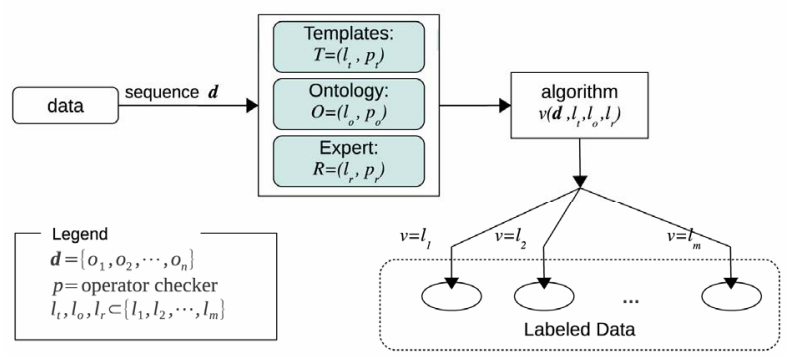

(a) Validators

\begin{tabular}{cc}
\hline Specifications & Examples \\
\hline field; type; repetition; missing & field; type; repetition; missing \\
$f_{1} ; f_{1}$-type; int;binary & ID; cmdid; $1 ;$ false \\
$f_{2} ; f_{2}$-type;int;binary & OPTION;option; $1 ;$ true \\
$f_{3} ; f_{3}$-type;int;binary & INPUT;input; 2 ;rue \\
$f_{4} ; f_{4}$-type;int;binary & ARGUMENT;argument; 2 ;rue \\
$f_{4} ; f_{5}$-type;int;binary & COMMAND;command; 1 true \\
$f_{6} ; f_{6}$-type;int;binary & DESTINATION;destination; $1 ;$ true \\
\hline
\end{tabular}

(b) Template Specification

Figure 4. Sequence validation.

expert. Ontological and expert operators are case and domain dependent and therefore are described further with a case study in Section 4. The different validation results $\left(l_{t}, l_{o}\right.$ and $\left.l_{r}\right)$ are then combined by using Algorithm 1. 
Algorithm 1. Aggregation of validation results.

\begin{tabular}{l}
$l \leftarrow l_{t}$ \\
if $l_{o} \neq$ null then \\
$\quad l \leftarrow l_{o}$ \\
end if \\
if $l_{r} \neq$ null then \\
$\quad l \leftarrow l_{r}$ \\
end if \\
\hline
\end{tabular}

Templates, as shown in Figure 3, are made up of all possible sequences of the desired NHCE and are in RDF format. Sequences in a template can be manually written or auto-generated using specifications shown in Figure 4(b). They are defined in terms of fields and their types with either missing occupants or entities, as defined in a definition files to which the types act as pointers, with a specified level of allowed repetition. For instance, with ssh commands as desired NHCE extracts, the first field (ID) can only have cmdid as its occupant with allowed repetition 1. The third field (INPUT), however, can be occupied by either of the following; null, input, input input etc. i.e. It is possible to have multiple inputs occupying an INPUT field (allowed repetition 2).

Each sequence in a template is assigned an observation score called sequence frequency-inverse page frequency, $s f$-ipf (Equation (1)) which shows how significant it is. It is denoted as $s$ in Figure 3. In Equation (1), $\|P\|$ is the total number of pages seen. For a document-based source like the Web, $\|P\|$ is the total number of documents. $s f(q, t)$ is the sequence count in a template $t$, (has value 1 in this proposed approach). The definition of sf-ipf is given by Definition 6. sf-ipf is analogous to tf-idf ([22]) which is a well known numeric statistic used in Information Retrieval and Text Mining for showing how important a word is in a document. In templates, there is also a provisioning for defining a bias weight based on the source of the extracts because for some sources (e.g. the Web), an extract can have a statistical advantage over others due to factors other than its information richness (e.g. social, commercial factors). Bias weights are specified using an approximated extracts distribution.

$$
\begin{aligned}
& s f-\operatorname{ipf}(q, t, P) \\
& =s f(q, t) \times \log \|P\|\|1\|+\{p \in P: t \in p\}
\end{aligned}
$$

$s f$-ipf is a numerical statistical weight on a sequence $(q)$ of types that reflects the significance of that sequence across all sequences (in a template $t$ ) which defines an extract.

\subsubsection{Approximated Distribution}

When parsing and matching an $n$-entity NHCE extract, we use $n$-bit binary flags to represent its matches whereby there are $2^{n-1}-1$ different flags. In ssh command exam- ple, where extracts are 6-entity as described in Section 2, there are $2^{5}-1=31 \quad(100000$ to 111111$)$ different flags. Since it is a complex problem to model how sequences are distributed across a heterogeneous information source (e.g. the Web) [16], our approach involves approximating this distribution using a minimum of information. We use the flag which corresponds to the commonly used extract occurrence. We approximate a distribution from this flag value (as the mean), using lognormal distribution (Equation (2)) under the following two assumptions.

Assumption 1: Extract occurrences are skewed distributed around a commonly used one. e.g. ssh-X user@ host

Assumption 2: The frequency of occurrence decreases with matched sub-entities. For instance, it is more likely to encounter ssh user than ssh-F file.txt user@host.

$$
\begin{aligned}
D(x)= & \exp \left(-(\ln x)^{2} /\left(2 \times \sigma^{2}\right)\right) x \\
& \times \sigma \times \sqrt{2 \pi}: x \leq 0 ; \sigma>0
\end{aligned}
$$

Standard deviation $\sigma$ provides a means to tune the distribution skewness depending on the desired extracts and respective source.

\subsubsection{Validation}

Validation is in two stages. The preliminary one is during template matching (Figure 3) and the other stage is during validation and output generation. Sequences are validated using a series of validation operators, the first of which is statistical, based on sf-ipf. A sequence is considered valid and assigned a label $l_{t}$ after its $s f$-ipf has passed a threshold frequency. Other case-specific operators are then enforced and labels $l_{o}$ and $l_{r}$ are assigned and then the various validation results are aggregated using a simple algorithm (Algorithm 1).

\subsubsection{Role of Sequence Validation}

In this approach, sequence validation plays two major roles. First is to refine the just extracted sequences of sub-entities by filtering out those which do not comply with the template through a matching process. And second is to validate template sequences using statistical $s f$-ipf and other user operators (ontological and direct expert input).

\subsection{Entity Transformation}

To guide transformation of an entity, we first characterize its field and then use support of these characteristics to produce a transformation model. We statistically characterize entities using their observed structures (length and count) and types (b-type and u-type). The used structural properties and types are shown in Table 3. 
Table 3. Entity characteristics.

\begin{tabular}{cc}
\hline Category & Characteristics \\
\hline Structural & Length, count \\
Basic types (b-types) & Text, number, alphanumeric \\
User-defined (u-types) & Regular expression \\
\hline
\end{tabular}

During extraction and parsing, characterized features are formed for each entity field, out of its occurrences. These are in triplet form 〈name,value,support $\rangle$. Occurrence of "-L 127.0.0.1" in INPUT field of the ssh command could produce characteristics such as 〈"count", 2, $\left.S^{\prime}\left(c_{i}\right)\right\rangle$, 〈"btype", "alphanumeric", $\left.S^{\prime}\left(c_{i}\right)\right\rangle$, $\left\langle\right.$ "utype", "ip", $\left.S^{\prime}\left(c_{i}\right)\right\rangle$ etc. Support $S^{\prime}\left(c_{i}\right)$ of a characteristic $c_{i}$ is a fraction of characteristic's count $\operatorname{count}\left(c_{i}\right)$ to all occurrences $\sum \operatorname{count}\left(c_{j}\right)$ (Equation (3)).

$$
S^{\prime}\left(c_{i}\right)=\operatorname{count}\left(c_{i}\right) \sum \operatorname{count}\left(c_{j}\right)
$$

Using support values of the four categories of characteristics in Table 3 (length, count, b-type, and u-type), a set of fuzzy rules are produced to guide entity transformation. Transformation states and fuzzy conditions are shown in Figure 5 and Table 4 respectively. Conditions for the transitions in Figure $\mathbf{5}$ which are indicated by numbers are shown in Table 4 .

\section{Case Study}

In this section we demonstrate the proposed approach and discuss its performance. We use linux commands (ssh, find and shutdown) as desired extracts and demonstrate extraction and subsequent use in solving a command validation problem. In our case study, we treat command validation problem as a classification problem. We use our approach to extract commands from the Web, parse them, validate them and ultimately label them to produce training data for use in building a classification model. In discussing and presenting this case study, we focus on one command only (ssh) and present the results for others in discussion (Section 4.5).

\subsection{Implementation}

We implemented the approach under Java environment using tools which are shown in Table 5. Figure 6 shows the design schematic of the implemented system. The details of the implementation are as follows.

1) Template is implemented using RDF container sequences as shown in Figure 7. In a template, field types (e.g. option and destination) also act as a pointers to files which define sub-entities (e.g. ip, email address etc.). A template contained 12 ssh command sequences (shown in Table 7) which were auto generated using specifications shown in Figure 4(b). The shown bias weights and sf-ipf
Table 4. Entity transformation: Conditions.

\begin{tabular}{cl}
\hline Transition & \multicolumn{1}{c}{ Condition(s) } \\
\hline 1 & $\mathrm{~S}($ b-type $)>\mathrm{THR}_{\mathrm{b}}$ \\
2 & $\mathrm{~S}($ count $)>\mathrm{THR}_{\mathrm{c}}$ or $\mathrm{S}($ length $)>\mathrm{THR}_{\mathrm{l}}$ \\
& $\mathrm{S}($ u-type $)>\mathrm{THR}_{\mathrm{u}}$ \\
& Savg(length, count,type $)>\mathrm{THR}_{\mathrm{a}}$ \\
3 & $\mathrm{~S}(\mathrm{~b}$-type $)>\mathrm{THR}_{\mathrm{b}}$ \\
\hline
\end{tabular}

Table 5. Used tools and environments.

\begin{tabular}{ll}
\hline Tool & Usage \\
\hline Gate's ANNIE [23] & $\begin{array}{l}\text { Extraction of text fragments which constitute } \\
\text { commands }\end{array}$ \\
RDF & $\begin{array}{l}\text { Template implementation. } \\
\text { Entity transformation model, supervised } \\
\text { learning. }\end{array}$ \\
Meka [10] & Command reference, Benchmark list. \\
The web & Data source \\
\hline
\end{tabular}

Table 6. Sub-entity types of ssh command.

\begin{tabular}{|c|c|}
\hline Type & Regular expression \\
\hline ip & $([01] ? \backslash \mathrm{d} \backslash \mathrm{d} ?|2[0-4] \backslash \mathrm{d}| 25[0 \cdots$ \\
\hline host & $([\mathrm{a}-\mathrm{Z} 0-9 \backslash-\mathrm{l}]+.\backslash$. (com $\mid$ org $\mid \cdots$ \\
\hline hostport & $(([01] ? \backslash \mathrm{d} \backslash \mathrm{d} ?|2[0-4] \backslash \mathrm{d}| 25[0 \cdots$ \\
\hline porthostport & $([1-9] \backslash \mathrm{d}\{0,3\})):(([01] ? \backslash \mathrm{d} \cdots$ \\
\hline hostporthostport & $(([01] ? \backslash \mathrm{d} \backslash \mathrm{d} ? \mid 2[0-4] \backslash \mathrm{d} \cdots$ \\
\hline user & {$[|w|-]([|| w])+.[\mid w]+@(([0 \cdots$} \\
\hline time & $([1-9]|(1[0-9])|(2[0-4])) \cdots$ \\
\hline directory & $\left((\backslash) ?[\wedge \mid s]+\backslash([\wedge \mid s]+\mid) ?\left(\left[{ }^{\wedge} \cdots\right.\right.\right.$ \\
\hline file & $\left(\left((\backslash) ?\left[{ }^{\wedge} \mid \mathrm{s}\right]+\backslash([\wedge \mid \mathrm{\wedge}]+1) ?\left(\left[{ }^{\wedge} \cdots\right.\right.\right.\right.$ \\
\hline tun:tun & $([1-9] \backslash \mathrm{d}\{0,2\})):([1-9] \backslash \mathrm{d}\{\cdots$ \\
\hline
\end{tabular}

Table 7. Comma separated ssh template sequences.

\begin{tabular}{clcc}
\hline no. & Sequence & $\mathrm{w}_{\mathrm{b}}$ & sf-ipf \\
\hline 1 & cmdid,null,null,null,null,null & 0.68 & 0.002 \\
2 & cmdid,null,null,argument,null,null & 0.5 & 0.002 \\
3 & cmdid,null,input-input,null,null,null & 0.36 & 0.004 \\
4 & cmdid,null,input,null,null,null & 0.36 & 0.004 \\
5 & cmdid,null,input-input,argument,null,null & 0.25 & 0.146 \\
6 & cmdid,null,input,argument,null,null & 0.25 & 0.058 \\
7 & cmdid,option,null,null,null,null & 0.18 & 0.005 \\
8 & cmdid,option,null,argument,null,null & 0.13 & 0.010 \\
9 & cmdid,option,input-input,null,null,null & 0.09 & 0.291 \\
10 & cmdid,option,input,null,null,null & 0.09 & 0.024 \\
11 & cmdid,option,input-input,argument,null,null & 0.07 & 0.291 \\
12 & cmdid,option,input,argument,null,null & 0.07 & 0.058 \\
\hline & & &
\end{tabular}




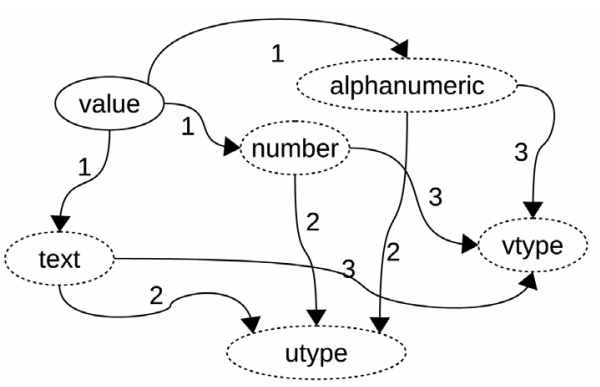

Figure 5. Entity transformation: States.

scores are after extraction and validation and are therefore described in Section 4.3.
2) Entity Type Definitions are shown in Table 6. We defined 10 utypes used in ssh commands.

3) Generic Rules, although seem complicated as shown in Figure 8, are easy to write because the details of subentity relationships are ignored at this stage. The rules do not cater for COMMAND field of ssh command.

4) Lookup Lists provide references to various NHCE keywords like cmdid (e.g. ssh), options (e.g. -l) etc.

5) Characterized Features give a statistical snapshot of observed sub-entities in the fields. Table 8 shows characteristic triplets in tabular form. The values in the table are discussed in Section 4.3.

6) Transformation Model is implemented using C 4.5

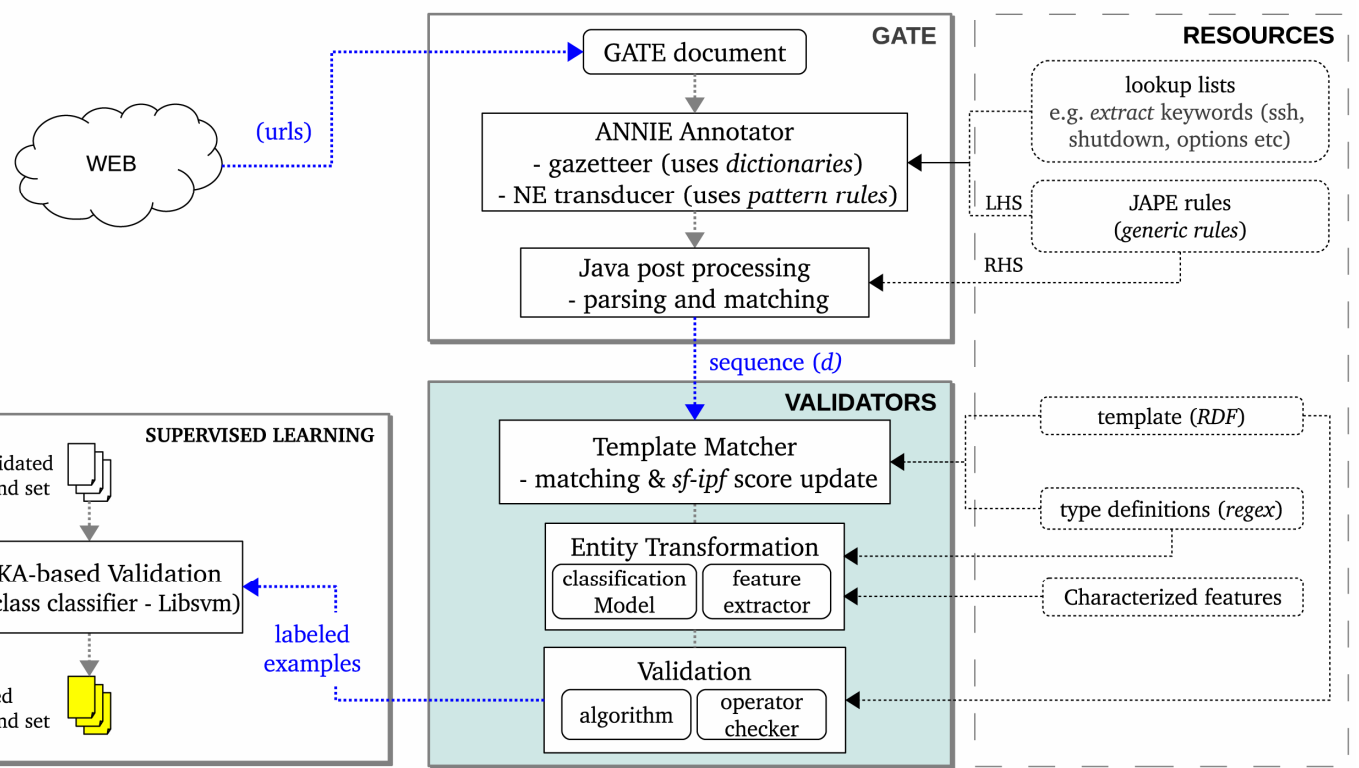

Figure 6. Schematic of the implemented design.

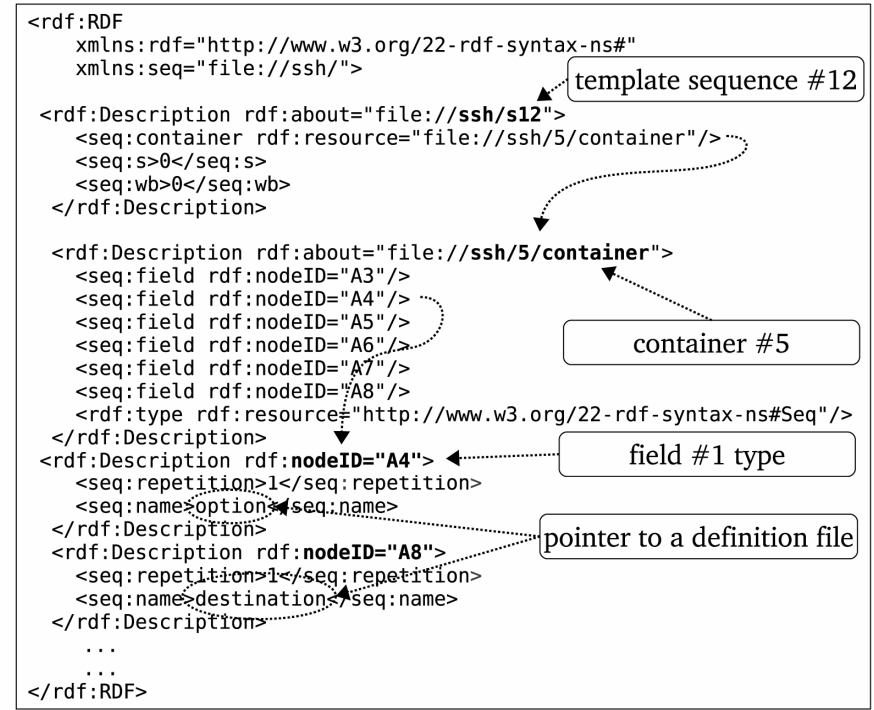

Figure 7. Ssh template. 


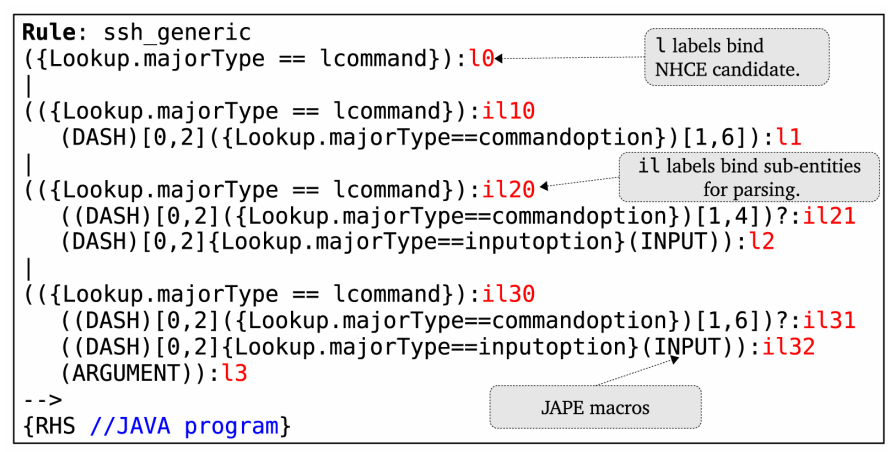

Figure 8. JAPE pattern rule for ssh commands.

Table 8. Field characteristic triplets in tabular form.

\begin{tabular}{cccccc}
\hline \multirow{2}{*}{ Characteristic } & \multirow{2}{*}{ Value } & \multicolumn{5}{c}{ Field support } \\
\cline { 3 - 6 } count & & $\mathrm{S} 1$ & $\mathrm{~S} 2$ & $\mathrm{~S} 3$ & $\mathrm{~S} 4$ \\
\hline count & 2 & 0.00 & 0 & 0.1 & 0.00 \\
count & 1 & 1 & 1 & 0.9 & 0.98 \\
length & $\cdot$ & $\cdot$ & $\cdot$ & $\cdot$ & $\cdot$ \\
length & 2 & 0.00 & 0.91 & 0.33 & 0.27 \\
length & 1 & 0.00 & 0.09 & 0.03 & 0.00 \\
b-type & alphanumeric & 0.00 & 0 & 0.09 & 0.05 \\
b-type & text & 1 & 0.85 & 0.81 & 0.95 \\
b-type & number & 0.00 & 0.15 & 0.1 & 0.00 \\
u-type & host & 0.00 & 0 & 0.02 & 0.09 \\
u-type & port & 0.00 & 0.12 & 0.09 & 0.00 \\
u-type & port-host-port & 0.00 & 0 & 0.06 & 0.00 \\
u-type & user & 0.00 & 0 & 0.00 & 0.55 \\
u-type & directory & 0.02 & 0.00 & 0.07 & 0.00 \\
\hline
\end{tabular}

algorithm which is built out of manually crafted fuzzy rules based on transitions shown in Figure 5.

7) Only template operator is used because we feel that the rationale behind any ontology and direct expert input is ambiguous for evaluational purposes.

\subsection{Experimentation Data}

We retrieved from the Web $120 \mathrm{html}$ pages which contain information (usage and example) about ssh command, $50 \mathrm{html}$ pages for shutdown command and 70 for find command. We used queries like "how to use...", "linux command reference", "how to connect using...", "how to shutdown...", "how to locate...", "how to find..." etc. against search engines and retrieve relevant pages.

\subsection{Extraction, Validation and Results}

This section and Section 4.4 are discussed using ssh command. Documents were annotated using GATE's
ANNIE annotator in which we used our generic rules. Annotation results depicted that the documents contain about 959 ssh command candidates (extracts) with which we experimented. Our objectives were to investigate:

- Effectiveness of our post-rule processing in extracting and parsing NHCE.

- Performance of sf-ipf based validation method.

- Effects of bias weights.

- How rich the current Web content is in terms of diversity and utility of its extracts.

To realize these objectives, we manually prepared a benchmark list of 1250 valid ssh commands. We do not claim that this is a comprehensive list of ssh commands. The list only covers ipv4, does not include neither COMMAND field nor redirections and pipelines (for simplicity and easiness in experimentation). Threshold values were set proportionally, i.e. by assuming even distribution across characteristic values with the exception to structural characteristics (count and length) which were set to match btype characteristics. For instance, since there are 3 b-types, then $T H R_{b}=30 \%$. Other thresholds are as follows, $T H R_{c}=T H R_{l}=30 \%, T H R_{u}=$ $10 \%$ and $T H R_{a}=60 \%$. Threshold $T H R_{\text {sf-ipf }}$ was set to the template average value of sf-ipf. The values of bias weights used were changing depending on the observed most common sequence. In the end, the bias weights were as shown in Table 7 with sequence No. 2 as the most common sequence, and therefore used a mean in the distribution Equation (2).

\subsubsection{Post-Rule Processing Results}

Post-rule processing serves as both a parser and initial validator. Out of the 959 candidate extracts, 275 were validated at this stage as to contain ssh commands. These were contained in 56 of the 120 used documents, meaning that the positive predictive value (PPV) of Web retrieval was $47 \%$. The distribution of extracts among documents is such that a document contains 4.9 extracts on average, document with the highest number of extracts has 35 extracts and the majority of documents contain only 1 extract. The validated candidates were 
also parsed into NHCE sub-entities for each of which its occurrences were used in characterizing that field. Table 8 summarizes characterized feature triplets and their observed support values.

\subsubsection{Validation Results}

In the template, 9 of the 12 template sequences were validated using $s f$-ipf. These resulted into 171 extract of which 70 successfully transformed. For example, ssh- $R$ 5555: localhost: 3389 to ssh- $R$ porthostport is a successful transformation while ssh-R 5555: localhost: 3389 to $s$ h- $R$ alphanumeric is a failed transformation. A field transformation into a non-existing type (neither utype nor vtype) is considered a failure and renders the whole extract sequence transformation unsuccessful. Like indicated in Figure 5, b-type is therefore not a final transformation state. Table 9 summarized the results of transformation for each field.

When checked against the benchmark list, the precision of the successfully validated and transformed extracts was $83 \%$ while recall was $27.46 \%$.

\subsubsection{Effects of Bias Weights}

Bias weights are meant to enable user to reduce the effect of Web-inherent statistical advantage. By changing the value of $\sigma$ (Equation (2)), the significance of a template sequence can be tuned. Without bias weights, 9 out of 12 template sequences were validated as pointed out earlier. With $\sigma=0.5$ bias however, sequence No. 6 is invalidated (Figure 9).

Table 9. Entity transformation summary.

\begin{tabular}{ccccc}
\hline Field & $f_{1}$ & $f_{2}$ & $f_{3}$ & $f_{4}$ \\
\hline Occurrences & 2 & 18 & 42 & 55 \\
u-type & 1 & 0 & 10 & 46 \\
v-type & 1 & 14 & 3 & 7 \\
Rate (\%) & 100.0 & 77.80 & 31.0 & 96.4 \\
Precision (\%) & 100.0 & 100.0 & 76.9 & 86.8 \\
Recall (\%) & 100.0 & 58.3 & 45.5 & 100 \\
\hline
\end{tabular}

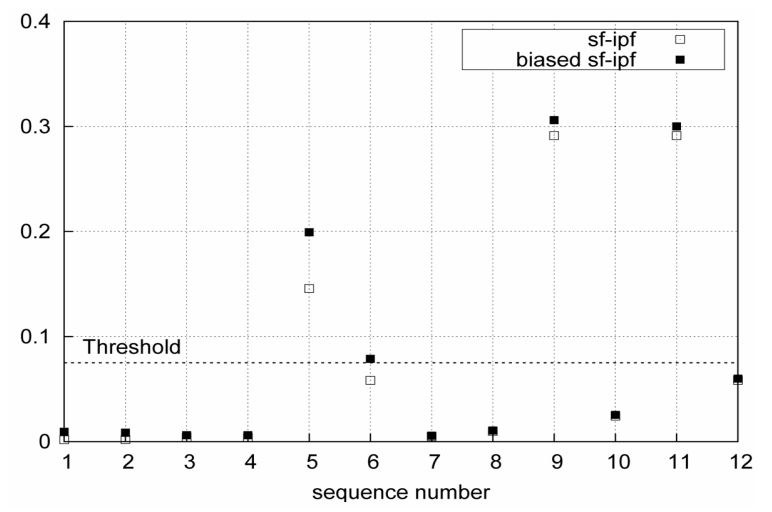

Figure 9. Biasing sf-ipf.

\subsection{Supervised Learning}

The final validated and transformed extracts comprise of sequences of vtypes and utypes (e.g. ssh-v-l user host). These can be used for further validation of ssh commands through either direct use as templates or as labeled examples in supervised learning. We experimented the later by using v-types and utypes as attributes in training datasets and build one class svm classifiers for validation of ssh commands. By using a one class classifier, invalid commands are considered outliers. 10-fold cross validation results, using the benchmark list as testing dataset, depicted a classifier with F-Measure 0.336 and accuracy of $39.33 \%$.

\subsection{Discussion}

The presented case study demonstrates our proposed approach in terms of how NHCE candidates are annotated from free text, parsed, validated, sub-entity transformed and finally used in validating similar NHCEs in a supervised learning setup. Table $\mathbf{1 0}$ summarizes results for all three linux commands which were experimented.

Our rule-based annotation approach is rather of a brutal force nature because relationships between subentities were not taken into account when writting the general rules. The initial extracts were therefore too noisy. For instance a text fragment ssh $-X-L$ 172.0.0.1 hostmachine led to two extract candidates with both $-L$ 172.0.0.1 and hostmachine considered as $f_{3}$ occurrences in one and $-L 172.0 .0 .1$ as $f_{3}$ occurrence while hostmachine as $f_{4}$ occurrence in another. Nonetheless the approach is preferred for a number of reasons in addition to existing facts like rule-based methods being methods which offer sound performances as pointed out in Section 2. First reason is that generic rules can be easily written and interpreted. Second reason is that, by considering all ambiguous text fragments in contention, fields in a template sequence are able to see more occurrences and so improve the richness of their entity

Table 10. Results summary.

\begin{tabular}{ccccc}
\hline Item & Metric & ssh & Shutdown & Find \\
\hline Annotation & No. of extracts & 959 & 77 & 276 \\
Post processing & No. of extracts & 275 & 59 & 215 \\
Doc. retrieval & PPV & 47.0 & 75.3 & 31.2 \\
Transformation & Success rate & 40.9 & 88.2 & 46.2 \\
& No. of extracts & 171 & 59 & 186 \\
Template operator & Precision & 83.0 & 93.2 & 63.2 \\
& Recall & 27.5 & 34.0 & 9.0 \\
Supervised & No. examples & 70 & 52 & 86 \\
learning & Accuracy & 39.3 & 45.9 & 17.4 \\
& F-measure & 0.3 & 0.4 & 0.1 \\
\hline
\end{tabular}


characteristics. Improved entity characteristics lead to better entity transformation. In Table 9, for example, although only 10 different vtypes appear in the final validated extracts, 14 were transformed.

The use of hand-coded binding labels for parsing falls in the same line of reasoning as generic rules. These are equally important in order to maximize field observations in a top-down extraction approach.

The initial validation, during template matching, removes noisy extracts, a consequence of using generic rules. As presented, initial validation saw candidate $e x-$ tracts reduced to 275 from 959 for ssh command. This is a $71 \%$ noise. shutdown and find do not appear too noisy as shown in Table 10 (23.4\% for shutdown and 22.1\% for find) because of the relatively few documents used and lesser popularity within the Web content.

sf-ipf based validation approach have shown both strength in filtering valid transformable extracts. This is supported by good precision values for all three commands (ssh-83.0\%, shutdown-93.2\% and find-63.2\%). These results might demonstrate neither richness of Web content nor practical utility of the extracts because of very low recall values but demonstrate the effectiveness of sf-ipf in filtering out best NHCE candidates from the original extracts. From our experimentations, it is evident that sf-ipf alone does not produce comprehensive enough results for practical use. This is depicted by low accuracy values when the extracts were used in supervised learning. It is a result of being only statistical and the use of generic rules. For instance, since an occurrence shutdown $-c$ is often then an extract shutdown-c now is likely to be validated even though it is not valid. As pointed earlier, it is for this reason that our approach incorporates expert input in either ontological operators or direct input operators forms. These operators are provided in terms of relationships between sub-entities. For example, an ontology or list which describes all valid inputs for a particular input flag in ssh commands. Surely, when used, these improve performance of extracts. When we experimented using direct input by specifying all valid inputs for the most observed input flag $(-L)$ in ssh command, we noticed a $10 \%$ increase in supervised learning accuracy.

Another reason for low accuracy values of the end classification models is the small number of used documents relative to what is avaliable in the Web. Preparation of experimentation documents was done manually, therefore it was quite an expensive process.

\section{Related Works}

Although Bibpro [1] is a citation parser, it resembles our work in the use of sequence templates. In Bibpro, structural properties and local properties of citation fields are used to create template sequences. This means that a significant prior knowledge about these properties is needed in advance. In our approach, template sequences are created from user's specifications about sub-entity types which occupy NHCE field. Also Bibpro's use of canonicalization makes it highly reliant to NLP. In terms of cardinality of sub-entities, "Booktitle" and "Month" are the fields of highest cardinality (12) which are nevertheless made up of only NPL keywords. We could not compare our approach against Bibpro because of differences in extract types and difficulty in implementation of Bibpro as its not open source.

The idea of using relational databases [5] can also be applied in recognition and classification of NHCEs. According to this idea, extracts are considered as database queries which have well understood formats and relationships between sub-entities (columns) are not explicit. Although in [5], this idea is presented in NPL context, it provides food for throught in dealing with NHCE.

Transformation of sub-entities is closely related to the discovery of new attributes. Wong et al. [2] employ semantic analysis on text fragments and use Bayesian Learning to infer them. The approach enables discovery of such attributes like "online price", "publishing year" etc. using EM algorithm model.

Statistical methods using state-of-the art HMM [13] and CRF [16] are promising ones in dealing with NHCEs. The challenge, however, lies in making them easy to deploy and understand, and integrate with expert input.

To the best of our knowledge, there is no published IE work for the extraction and classification of NHCEs. Most studied extracts are made up of keyword subentities for which dictionaries [8] and lists are preferred.

\section{Conclusions}

This paper presented an approach to recognize and classify Named High cardinality Entities. The approach treats sub-entities of a NHCE as a extract sequence and uses a series of three categories of operators to validate these extract sequences. The foundational operator is a template based operator which scores template sequences with a statistical score called sequence frequency-inverse page frequency and use these sequences to validate $e x-$ tract sequences. The approach also enables sub-entities to transform into either a value type (v-type) or a userdefined type (u-type). This transformation brings flexibility on how extraction knowledge is shared, how extract features are captured and also how extracts are used.

We have demonstrated this approach using linux commands as desired NHCEs. Experimentation results reveal the effectiveness of sequence validation based extraction in capturing both structural and user-defined features and types, characterize them and subsequently use the characterized features to validate extract sequences. We have 
also shown how an approximated distribution can be used to tune significances of template sequences as a way to regulate statistical acceptability of extract sequences.

There are some areas where improvements are still needed in order to make this approach easier to use and deploy. These includes automating the process of building a transformation model, improving the generic rules approach so that extracts are less noisy but in sufficient numbers and developing a framework by which ontological operators can be smoothly integrated. Other issues, which are to be further researched on, concern alternatives to generic rules approach in annotating and parsing NHCE extract, e.g. statistical CRF-based.

\section{Acknowledgements}

A part of this work is supported by third supplementary budget for 2011 of Ministry of Internal Affairs and Communications of Japan, Research and Development of Applicable Resource Unit Construction and Reconstitution Technology for Communication Network on Large-Scale Disasters.

\section{REFERENCES}

[1] C. C. Chen, K. H. Yang, C. L. Chen and J. M. Ho, "Bibpro: A Citation Parser Based on Sequence Alignment," IEEE Transactions on Knowledge and Data Engineering, Vol. 24, No. 2, 2012, pp. 236-250.

doi:10.1109/TKDE.2010.231

[2] T. L. Wong and W. Lam, "Learning to Adapt Web Information Extraction Knowledge and Discovering New Attributes via a Bayesian Approach," IEEE Transactions on Knowledge and Data Engineering, Vol. 22, No. 4, 2010, pp. 523-536. doi:10.1109/TKDE.2009.111

[3] F. Ashraf, T. Ozyer and R. Alhajj, "Employing Clustering Techniques for Automatic Information Extraction from Html Documents," IEEE Transactions on Systems, Man, and Cybernetics, Part C: Applications and Reviews, Vol. 38, No. 5, 2008, pp. 660-673. doi:10.1109/TSMCC.2008.923882

[4] D. C. Wimalasuriya and D. Dou, "Components for Information Extraction: Ontology-Based Information Extractors and Generic Platforms," Proceedings of the 19th ACM International Conference on Information and Knowledge Management, New York, 2010, pp. 9-18. http://doi.acm.org/10.1145/1871437.1871444

[5] L. Tari, P. H. Tu, J. Hakenberg, Y. Chen, T. C. Son, G. Gonzalez and C. Baral, "Incremental Information Extraction Using Relational Databases," IEEE Transactions on Knowledge and Data Engineering, Vol. 24, No. 1, 2012, pp. 86-99. doi:10.1109/TKDE.2010.214

[6] S. A. Kripke, "Naming and Necessity," Harvard University Press, Cambridge, 1980.

[7] D. Nadeau and S. Sekine, "A Survey of Named Entity Recognition and Classification," Linguisticae Investiga- tiones, Vol. 30, No. 1, 2007, pp. 3-26. doi:10.1075/li.30.1.03nad

[8] J. L. Hong, "Data Extraction for Deep Web Using Wordnet," IEEE Transactions on Systems, Man, and Cybernetics, Part C: Applications and Reviews, Vol. 41, No. 6, 2011, pp. 854-868. doi:10.1109/TSMCC.2010.2089678

[9] P. McFedries, "The Coming Data Deluge [Technically Speaking]," IEEE Spectrum, Vol. 48, No. 2, 2011, pp. 19. doi:10.1109/MSPEC.2011.5693066

[10] I. H. Witten, E. Frank and M. A. Hall, Data Mining: Practical Machine Learning Tools and Techniques," 3rd Edition, Morgan Kaufmann, Burlington, 2011.

[11] S. Lawrence, C. L. Giles and K. Bollacker, "Digital Libraries and Autonomous Citation Indexing," IEEE Computer, Vol. 32, No. 6, 1999, pp. 67-71. doi:10.1109/2.769447

[12] J. Han, M. Kamber and J. Pei, "Data Mining: Concepts and Techniques," 2nd Edition, Morgan Kaufmann, Burlington, 2006.

http://www.amazon.com/Data-Mining-Concepts-Techniq ues-Management/dp/1558609016

[13] E. Agichtein and V. Ganti, "Mining Reference Tables for Automatic Text Segmentation," Proceedings of the 10th ACM SIGKDD International Conference on Knowledge Discovery and Data Mining, 2004, pp. 20-29.

[14] V. Borkar, K. Deshmukh and S. Sarawagi, "Automatic Segmentation of Text into Structured Records," 2001.

[15] R. Malouf, "Markov Models for Language-Independent Named Entity Recognition," Proceedings of the 6th Conference on Natural Language Learning, Stroudsburg, 2002, pp. 1-4. doi:10.3115/1118853.1118872

[16] C. Sutton and A. McCallum, "An Introduction to Conditional Random Fields for Relational Learning," 2006.

[17] M. Asahara and Y. Matsumoto, "Japanese Named Entity Extraction with Redundant Morphological Analysis," Proceedings of the 2003 Conference of the North American Chapter of the Association for Computational Linguistics on Human Language Technology, NAACL, Morristown, 2003, pp. 8-15. doi:10.3115/1073445.1073447

[18] O. Etzioni, M. Cafarella, D. Downey, A. M. Popescu, T. Shaked, S. Soderland, D. S. Weld and A. Yates, "Unsupervised Named-Entity Extraction from the Web: An Experimental Study," Artificial Intelligence, Vol. 165, 2005, pp. 91-134. doi:10.1016/j.artint.2005.03.001

[19] A. Yates, "Extracting World Knowledge from the Web," IEEE Computer, Vol. 42, No. 6, 2009, pp. 94-97. doi:10.1109/MC.2009.188

[20] H. Cunningham, D. Maynard and V. Tablan, "JAPE: A Java Annotation Patterns Engine (Second Edition)," Technical Report, University of Sheffield, Sheffield, 2000.

[21] S. Sarawagi, "Information extraction," Found Trends Databases, Vol. 1, No. 3, 2008, pp. 261-377. doi:10.1561/1900000003

[22] H. C. Wu, R. W. P. Luk, K. F. Wong and K. L. Kwok, "Interpreting tf-idf Term Weights as Making Relevance Decisions," ACM Transactions on Information Systems, 
Vol. 26, No. 3, 2008, pp. 1-37. doi: $10.1145 / 1361684.1361686$

[23] H. Cunningham, D. Maynard, K. Bontcheva and V. Tablan, "GATE: A Framework and Graphical Development
Environment for Robust NLP Tools and Applications," Proceedings of the 40th Anniversary Meeting of the Association for Computational Linguistics (ACL), Philadelphia, 7 February 2003. 